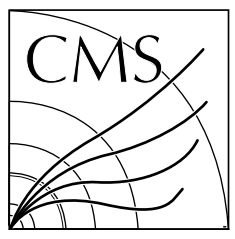

The Compact Muon Solenoid Experiment

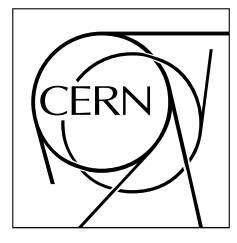

February 25, 2003

\title{
Cross-calibration of two automatic quality control systems for the CMS ECAL crystals
}

\author{
E. Auffray, E. Bouquerel, G. Chevenier, P. Lecoq, \\ L. Perez-Prado, P. Sempere-Roldan ${ }^{1}$ \\ CERN, Geneva, Switzerland \\ M. Schneegans \\ LAPP, Annecy-le-Vieux, France \\ M. Castellani, F. Cavallari, S. Costantini, I. Dafinei, \\ M. Diemoz, E. Longo, G. Organtini, R. Paramatti \\ Dipartimento di Fisica, Università "La Sapienza”, Roma, Italia \\ INFN, Sezione di Roma, Italia \\ S. Baccaro, M. Montecchi \\ ENEA Casaccia, Roma, Italia
}

\begin{abstract}
The barrel part of the CMS electromagnetic calorimeter consists of about 60000 Lead Tungstate (PWO) crystals and will be assembled in two Regional Centers, nearby Rome and at CERN. Two automatic machines have been designed to check the crystal quality before assembly. The main crystal characteristics are compared to a set of specifications included in the contract with the crystal producers. The stability and inter-calibration between the two machines is a fundamental issue, which has to be monitored during the whole construction phase. This paper describes the checks that were made in order to cross-calibrate the measurements and to guarantee a proper selection of crystals for the detector.
\end{abstract}

To be submitted to Nucl. Instr. \& Meth. A

\footnotetext{
${ }^{1}$ On leave from Universidad de Santiago de Compostela, Santiago de Compostela, Spain
} 


\section{Introduction}

The CMS collaboration at the LHC has decided to construct a homogeneous electromagnetic calorimeter made of about 80000 lead tungstate (PWO) crystals (see Ref. [1] and [2]). This choice has been driven by the search for the Higgs boson in the intermediate mass range $(100-140 \mathrm{GeV})$, where the most favorable decay goes via two high energetic photons. Thus, high resolution in energy and position for electromagnetic showers is required, to fight against irreducible background.

After a long R\&D period, the properties of the PWO crystals are now well adapted to the high radiation levels of the CMS environment and to the short beam crossing time of the LHC. To guarantee the high performance of the calorimeter the following properties have to be checked on each crystal before mounting in the experiment:

- the dimensions of the crystals have to be within very strict tolerances, in order to fit the mechanical structure avoiding any stress between crystals and to ensure maximal hermeticity of the calorimeter;

- $\quad$ the light yield (LY) of the crystals must be higher than a given threshold, this to ensure a small stochastic term in the energy resolution. The variation of the LY along the crystal length should not exceed a given value, to minimize its effect in the energy resolution of the calorimeter;

- the transmittance of the crystal along its longitudinal axis (LTO), in particular around $350 \mathrm{~nm}$, has been proven to be connected with the presence of defects which are correlated with the radiation tolerance of the crystals (see Ref. [3]). Based on this correlation, very strict requirements on the shape and level of the LTO were set. Furthermore the transversal transmission (TTO) of the crystals should not vary as a function of the position, since it would be a proof for crystal inhomogeneities.

All these requirements have been precisely stated in the contracts with the crystal producers; it is therefore of the outmost importance to be able to correctly measure these properties for every single crystal received.

\section{The ACCOS machines and crystal quality control procedure}

In the two Regional Centers for calorimeter assembly and test (one at CERN and one at ENEA near Rome), the staff thought about the design of a machine (called ACCOS machine) to rapidly measure the crystal properties. The ACCOS machines design principle is described in Ref. [4] and [5]. Since then, several machines were built in the two regional centers, two at ENEA Casaccia nearby Rome (see Ref.[6]) and four at CERN (see Ref. [7]).

Although the properties to be measured by the ACCOS machines at CERN and in Rome are the same, the measurement techniques used in the two are different. These differences help to avoid systematic biases in the measurements but on the other hand require careful checks. Given the required precision and the long time span during which the calorimeter will be assembled, a punctual protocol has been established in each center to ensure the stability of calibration and measures within the tolerances.

In the following, we describe the differences between the two machines, and the efforts made to intercalibrate them. We will concentrate the paper on the comparison of the optical and physics measurements and the light yield inter-calibration; as a matter of fact, the measurements of the crystal dimensions are performed by equivalent commercial 3D machines and therefore will not be discussed.

The crystals, whose measurements are discussed in this paper, are pre-production PWO barrel crystals. Crystals have the shape of truncated pyramids and one of the lateral faces is depolished in order to improve the light collection uniformity.

When received from the factory, crystals are visually inspected, cleaned and a barcode is glued on the small face, then they are loaded on special trays 5 by 5 . The ACCOS machines measure the crystals on the trays. The machines are equipped with a barcode reader, which, as a first step, reads the barcode of the crystals. The full set of measurements is performed automatically and the results are sent to the CRISTAL database (see Ref. [8]). 


\section{The measurement of the Light Yield and Light Collection}

Figure 1 shows the comparison between the light yield measured at CERN and in Rome.

The LY is important for its contribution to the stochastic term of the energy resolution of the calorimeter but also to the noise term. Crystals are accepted if their LY is higher than $8 \mathrm{pe} / \mathrm{MeV}$ when measured in reference conditions, defined as: at $18^{\circ} \mathrm{C}$, with a photomultiplier Philips XP2262B in optical contact with the large base of the crystal and the other surfaces wrapped with tyvek, as described in Ref. [9]. This threshold value corresponds to $4 \mathrm{pe} / \mathrm{MeV}$ when read with two APDs in the test-beam.

The required precision on the LY measurement is of the order of a few percent.

For what concerns the LY measurement, both centers use a radioactive source to excite the scintillating light driven by a motor to move the source along the crystal. Then for the light detection and evaluation of the LY, CERN is using the decay time method with integration of the spectrum (see Ref. [7]) and Rome is using the photo-peak detection, as described in Ref. [9].

The measurements take place in a dark room and care has been taken to eliminate any possible spurious light source.

The light signal itself is quite small, since the available radioactive sources with a long lifetime produce $\gamma$ rays of energies around $1 \mathrm{MeV}$ and PWO crystals have a rather low LY.

CERN uses a ${ }^{22} \mathrm{Na}$ source which is a $\beta^{+}$emitter. One of the two photons from the annihilation of the positron is detected by a scintillating crystal with a high LY (like $\mathrm{BaF}_{2}$ ) and the second one goes in the PWO crystal. The first crystal is used to trigger the data acquisition and reduce the background and to define the baseline time. In Rome, a ${ }^{60} \mathrm{Co}$ source is employed. It provides 2 photons of energy $1.17 \mathrm{MeV}$ and $1.33 \mathrm{MeV}$ respectively. The signal itself is used to trigger the data acquisition.

The effects that could spoil the comparison or introduce systematic differences are: a high noise or environmental background and differences between photomultiplier tubes, optical contacts and wrappings. Therefore all these points have been carefully studied.

It is to be mentioned here that the experimental setup of the ACCOS machines are different: at CERN there is no optical contact and no wrapping around the crystal, while the Rome laboratory has equipped the set-up with a tyvek wrapping to increase the light and the photomultiplier tube is kept at $1 \mathrm{~mm}$ distance from the crystal.

In both centers the LY is "normalized" to the measurement in reference conditions, as described above.

Both centers are equipped with so-called reference LY benches, where few crystals are used to periodically check the calibration of the machine and its stability. These measurements are done in reference condition.

All these careful procedures have finally given a very good agreement between the two reference benches, as Figure 1 shows. The threshold value is $8 \mathrm{pe} / \mathrm{MeV}$ and the achieved measurement precision is enough to guarantee a proper selection.

Figure 2 shows the distribution of the measured LY difference of the LY between the two laboratories. The standard deviation is: $\sigma(\mathrm{LY})=0.7 \mathrm{pe} / \mathrm{MeV}$ and the mean is $0.1 \mathrm{pe} / \mathrm{MeV}$, well within the resolution. The crystals, which are out of the specification in one center, will be re-measured before final rejection.

The crystals shape induces a focusing effect that causes light collection to be non uniform along the crystal. A high gradient in the Light Yield could spoil the calorimeter resolution, because the shower-toshower depth fluctuations could be sensitive to different light collection efficiencies. Production crystals are depolished on one face at the factory in order to decrease this effect and have a flatter light collection curve (see Ref. [10]).

We define the Front and Rear Non-Uniformities - FNUF and RNUF - respectively as the gradient of the LY with respect to the crystal length calculated between 3.5 and $11.5 \mathrm{~cm}$ and between 11.5 and $19.5 \mathrm{~cm}$ from the crystal front face, that is from the small base of the truncated pyramid. Reference [11] shows that there is a strong correlation between the single crystal energy resolution measured with a highenergy electron beam and the FNUF. The required limits on the FNUF are $\pm 0.35 \% / \mathrm{X}_{0}$, which correspond to an expected contribution of $0.3 \%$ to the constant term of the calorimeter energy resolution.

The precision achieved on the FNUF measurement is about $0.15 \% / \mathrm{X}_{0}$ in both laboratories. Figure 2 shows the comparison between the FNUF of some hundreds of crystals measured both in Rome and at CERN. We find satisfactory agreement between these measurements, within the accuracy of the measurement. As shown in Figure 2, the standard deviation of the difference between the crystal FNUF measured in the two laboratories is: $\sigma(\mathrm{FNUF})=0.19 \% / \mathrm{X}_{0}$, while the mean is centered at zero within the accuracy of the measurement. Crystals, which do not pass the requirement on the FNUF, are measured on the test bench of the CERN laboratory and their depolished face is further treated until the required FNUF range is reached. 

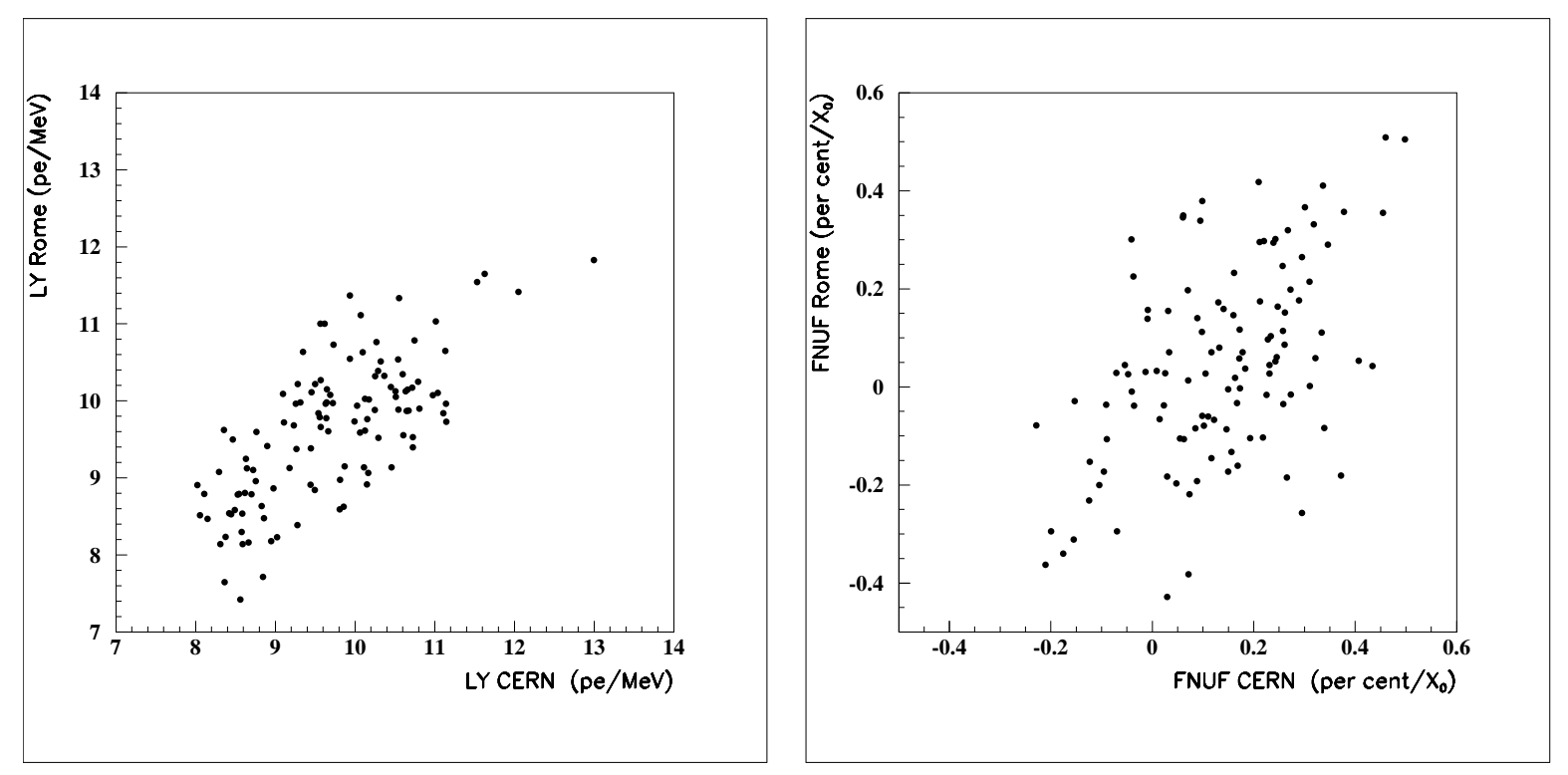

Figure 1: Comparison between the Light Yield (LY) and the FNUF measured by the ACCOS machines at CERN and in Rome for a few hundred crystals.
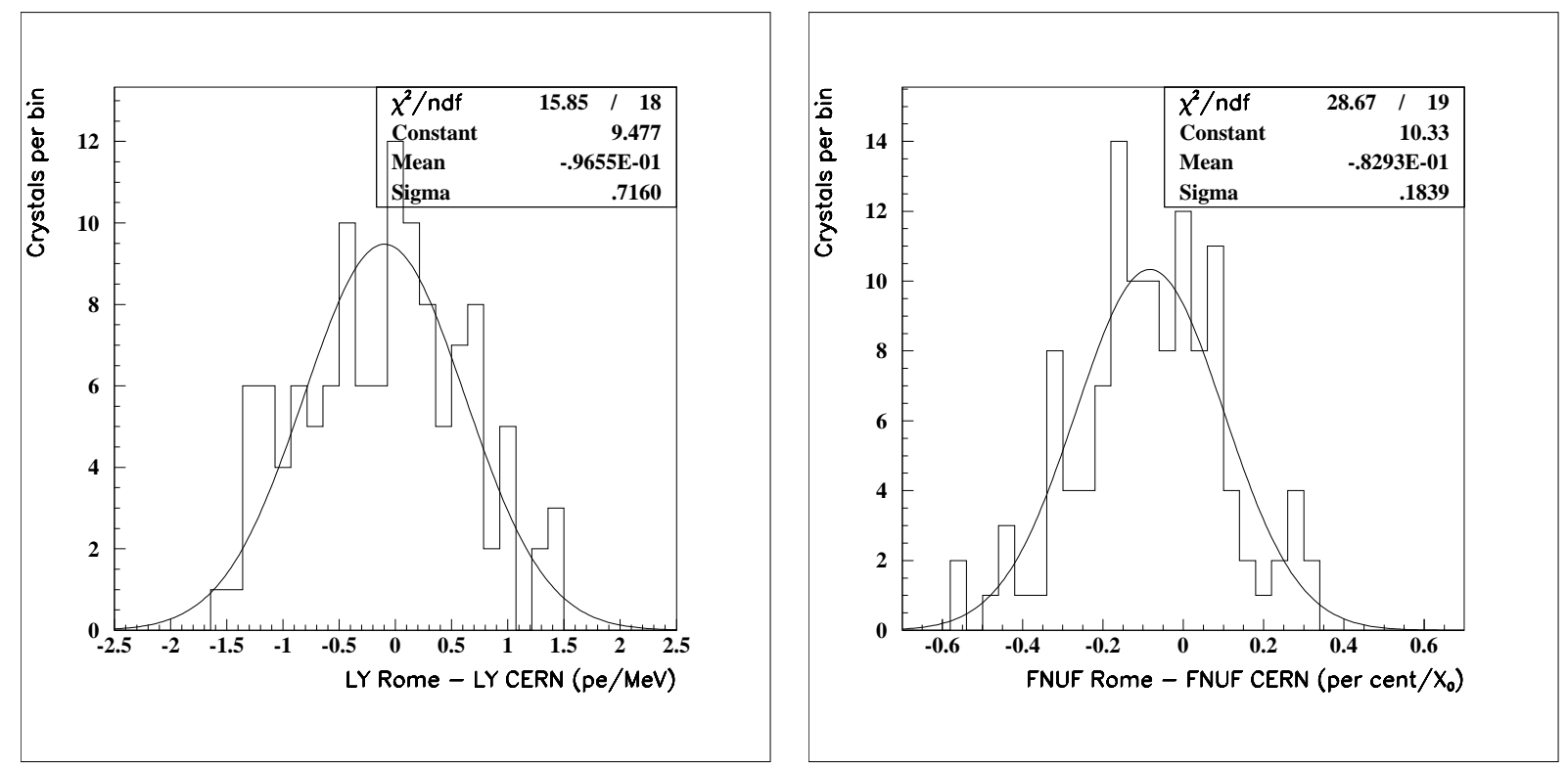

Figure 2: (right) Difference between the LY (left) and the FNUF (right) measured in Rome and at CERN for a few hundreds crystals. 


\section{The measurement of the Optical Transmission}

In Rome, Longitudinal and Transversal transmissions are measured through a system of mirrors with a single-beam CCD-diode array spectrophotometer (S2000 from Ocean Optics ${ }^{2}$ ) directly coupled to an integrating sphere (Labsphere of $150 \mathrm{~mm}$ diameter), as described in Ref.[6]. Data points are obtained every $4 \mathrm{~nm}$ from 300 to $700 \mathrm{~nm}$. At CERN Longitudinal and Transversal Transmissions are measured by two separate spectrophotometers using a large area photodiode as detector. The light wavelengths are selected using a set of narrow band pass filters nominally centered at: 330, 340, 350, 360, 380, 405, 420, 450, 492, 620 and $700 \mathrm{~nm}$.

Given the difference in the methods, at the beginning a small disagreement between the two set-ups was found, due to the integrating sphere and the different optical paths. In fact, the Rome set-up efficiently collects the light scattered from any possible crystal defect (bubbles, cracks and impurities) while the CERN one does this only partially. Normally a simple visual inspection was sufficient to identify the problem. However, crystals from recent batches do not have anymore such kind of defects.

Figure 3 shows the spectra of Longitudinal (LTO) and Transversal Transmissions (TTO) of a full length CMS PWO crystal. The LTO curve shows a plateau at long wavelengths and a sharp decrease below about $400 \mathrm{~nm}$, due to the band edge absorption. This sharp decrease is typical of radiation-hard PWO crystals. On the contrary, crystals that show a less steep descent are not good from this point of view. Quantitatively a parameter that has been found to be correlated to the radiation hardness is the slope at the inflection point (see Ref. [12]).

The Transmission at three crucial wavelengths $(360 \mathrm{~nm}, 420 \mathrm{~nm}$ and $620 \mathrm{~nm})$ give an indication of the optical quality of the crystal and are used as selection criteria, as described in Ref [12].

It has been suggested in Ref. [13] to fit the experimental points of the Transmission curve with the function:

$$
T(\lambda)=e^{-e^{-\left(\lambda-P_{0}\right) \cdot P_{1}} \cdot P_{2} \cdot\left(1-P_{3} \cdot e^{-\lambda / P_{4}}\right)}
$$

which describes well the range between $340 \mathrm{~nm}$ and $420 \mathrm{~nm}$. This function having too many parameters to be easily used with the few points measured by the CERN machine, we have reduced the number of free parameters by keeping constant the parameter 44 to the value of $92 \mathrm{~nm}$, as proposed in Ref. [13].

A program written in Java code fits automatically the crystal spectrum using several minimization techniques and estimates the fit parameters, the inflection point and the slope at the inflection point. On the basis of the fitted parameters we can then calculate the transmission at the 3 chosen wavelengths. The fit takes into account the errors associated with the experimental points for the two machines and the actual filter wavelengths for the CERN machine.

Three sets of crystals were considered to test ACCOS: Batch 4 and 5 are rather old crystals, and a more recent batch is shown as well. These batches show rather different values for the parameters, because the old crystals had a different doping; so with this set of crystals we have been able to test the validity of the method over a wide range of the fit parameters. Figure 4 and Figure 5 show the correlation observed for the considered crystals measured with the CERN and Rome machines: the Transmission at 360, 420 and $620 \mathrm{~nm}$, the wavelength of the inflection point, and the slope.

The threshold values are:

$$
\begin{array}{ll}
- & \mathrm{LTO}(360 \mathrm{~nm})>25 \% \\
- & \mathrm{LTO}(420 \mathrm{~nm})>55 \% \\
\text { - } & \text { LTO }(620 \mathrm{~nm})>65 \% \\
\text { - } & \text { Slope at inflection point }>3 \% / \mathrm{nm}
\end{array}
$$

The resolution for these parameters is shown in Figure 6 and it is accurate enough to guarantee a proper selection of crystals: $\sigma(\operatorname{LTO}(360))=1.6 \%, \quad \sigma(\operatorname{LTO}(420))=1.1 \%, \quad \sigma(\operatorname{LTO}(620))=0.87 \%$, $\sigma($ Slope $)=0.15 \% / \mathrm{mm}$.

Crystals, which are at the limit for the Longitudinal Transmission at $360 \mathrm{~nm}$ and the Slope, undergo a direct irradiation test.

\footnotetext{
2 www.oceanoptics.com
} 


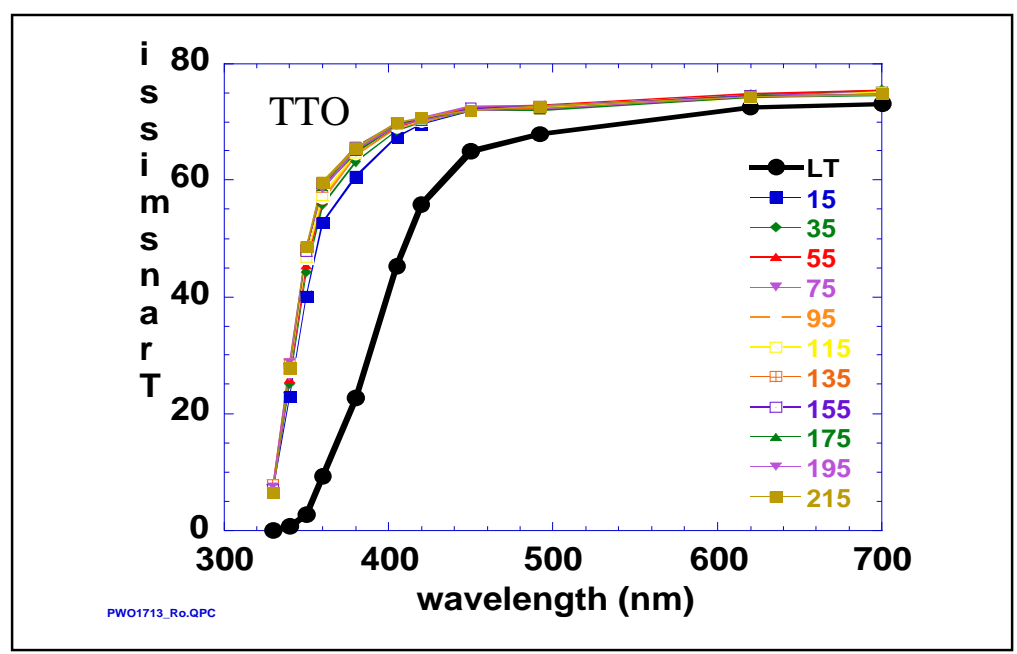

Figure 3: Longitudinal (LTO) and Transversal (TTO) transmission spectrum of a typical PWO crystal. Several positions are shown for the TTO measurement, from 15 to $215 \mathrm{~mm}$ from the front face of the crystal.
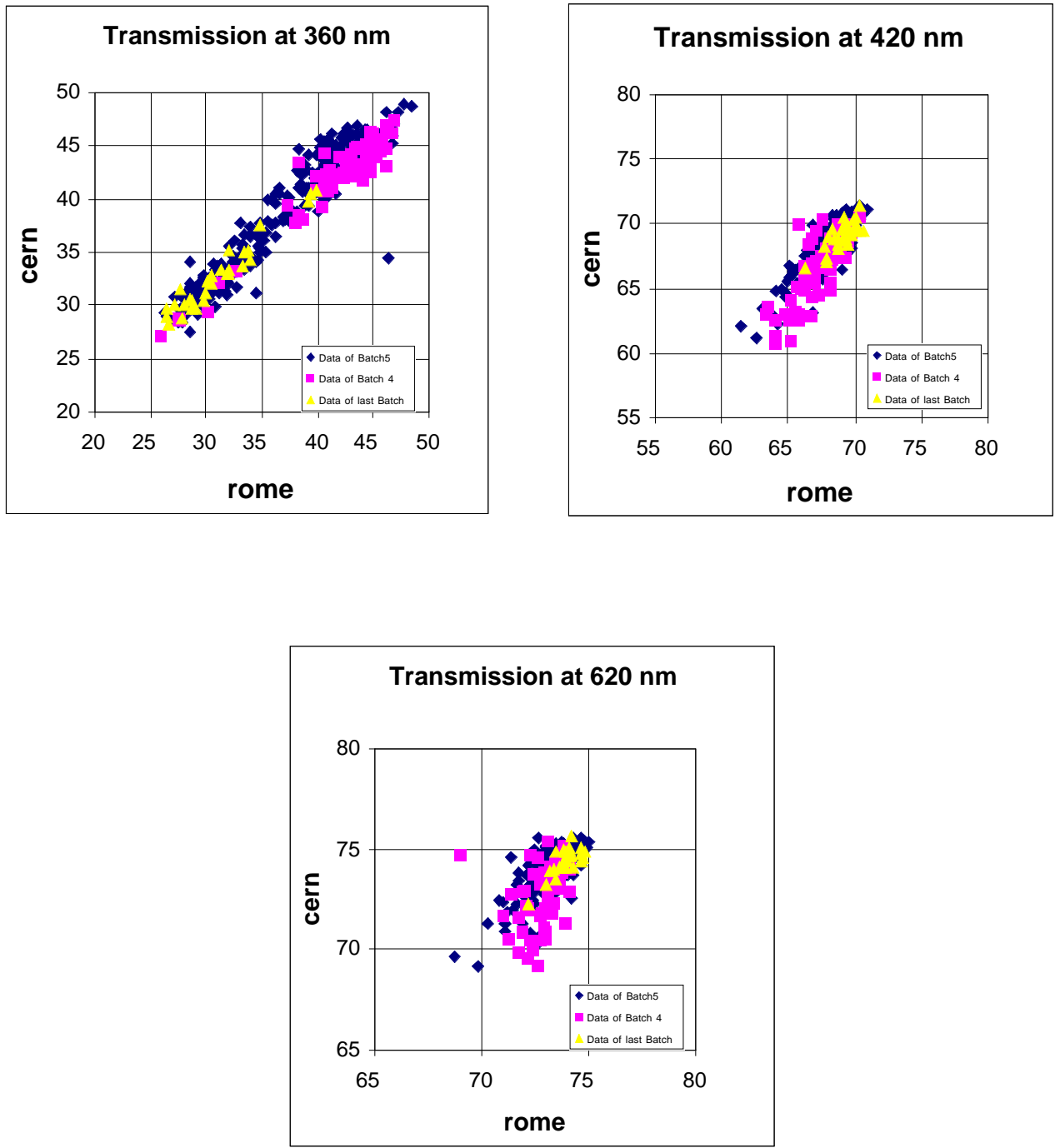

Figure 4: Longitudinal Trasmission: comparison of the Transmission as measured at CERN and in Rome for 3 wavelengths. The data are given in \%. 

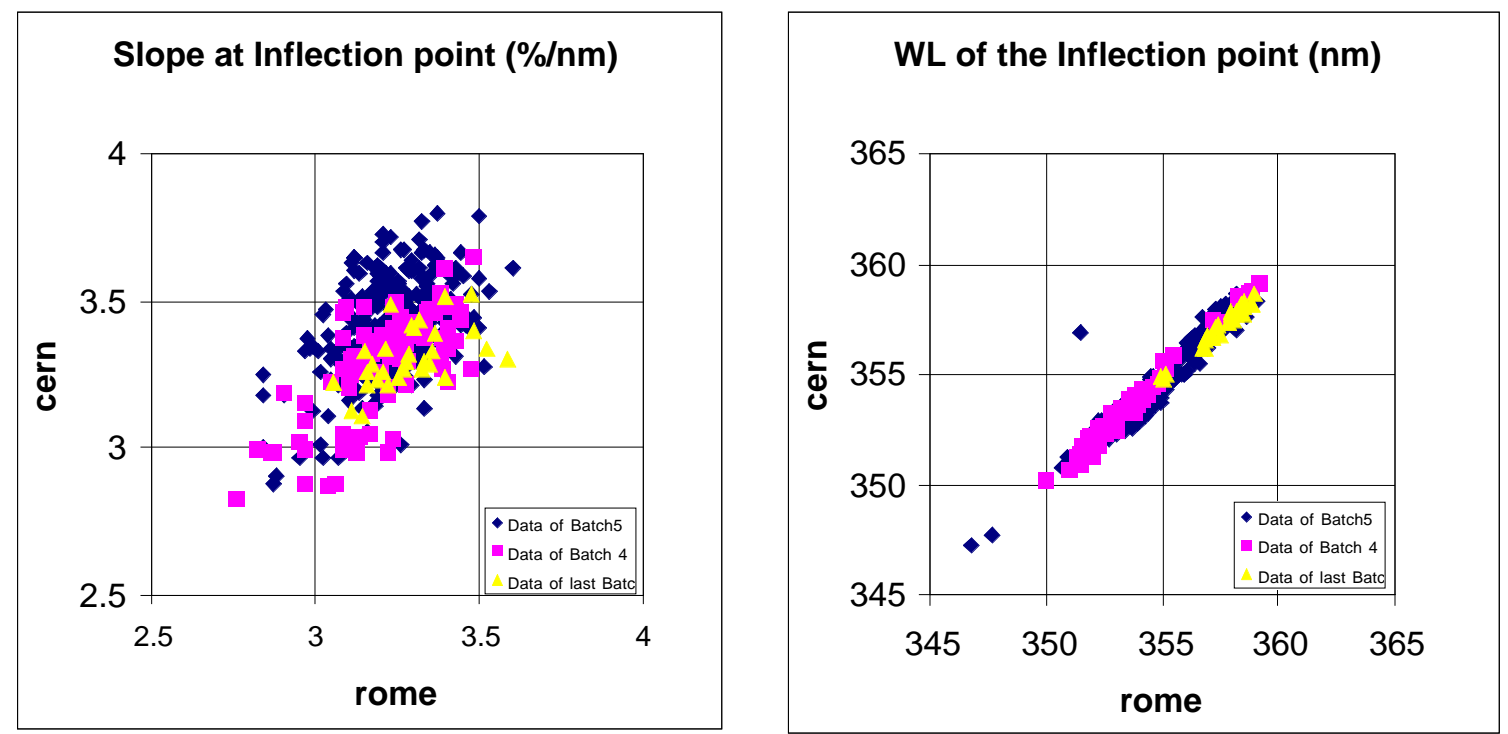

Figure 5: Slope of the LTO curve at inflection point and wavelength of the inflection point for 3 batches of crystals as measured in Rome and at CERN. The two sets of measurements are in good agreement with each other.
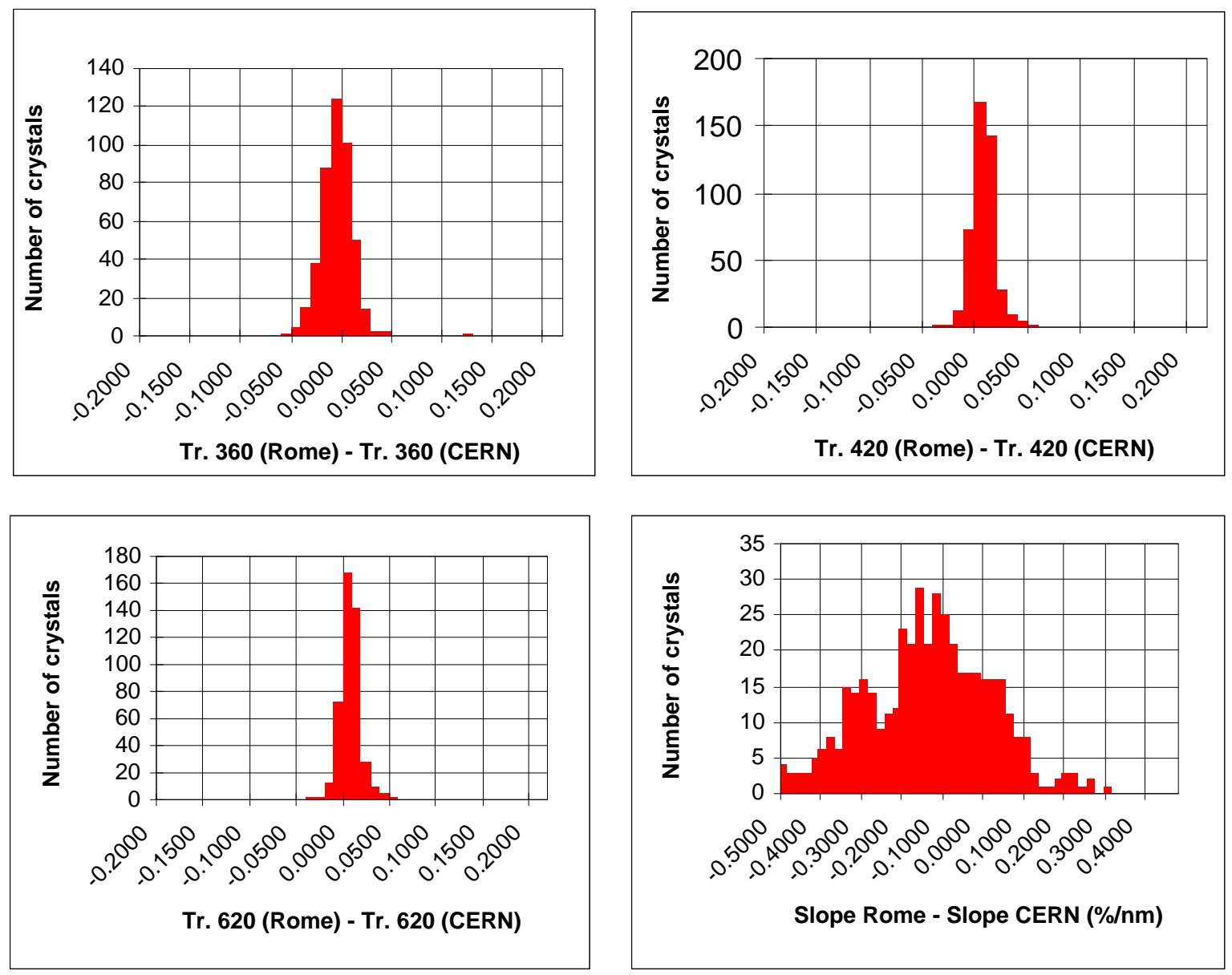

Figure 6: Difference between the Rome and the CERN measurements for the 4 parameters used for the crystal selection in the LTO: LTO at $360 \mathrm{~nm}, 420 \mathrm{~nm}, 620 \mathrm{~nm}$ and the Slope at the Inflection point. 


\section{$5 \quad$ The Transversal Transmission}

Figure 3 also shows typical Transversal Transmission spectra (TTO) for various positions along a crystal. This measurement is crucial to guarantee the homogeneity of the crystal properties along its length and also to evaluate the possibility to have a uniform crystal with respect to the FNUF (see Section 0). Thus the dispersion of the transversal transmission is one of the parameters, which is used to select good crystals from bad ones.

In the ACCOS machines - both at CERN and in Rome - the Transversal Transmission is measured for 11 positions along the crystal. Quantitatively we fit the 11 spectra, as described in Section 0, and we interpolate the wavelength $\left(\lambda_{50}\right)$ for which the crystal has a transmission of $50 \%$. Then we calculate the dispersion of this parameter in the 11 positions as well as its gradient, which is its variation along the crystal.

Figure 7 shows the correlation between Rome and CERN data for these two quantities. There is good agreement between the two sets of measurements.

The parameter that is used for the selection is the wavelength dispersion, which must be smaller than 3 nm.

Figure 8 shows the difference between the Rome and CERN measurement of this variable. The resolution $\sigma$ is $0.46 \mathrm{~nm}$, which is sufficient to guarantee a proper selection. The crystals, which do not pass this criterion, will not be used for the calorimeter.
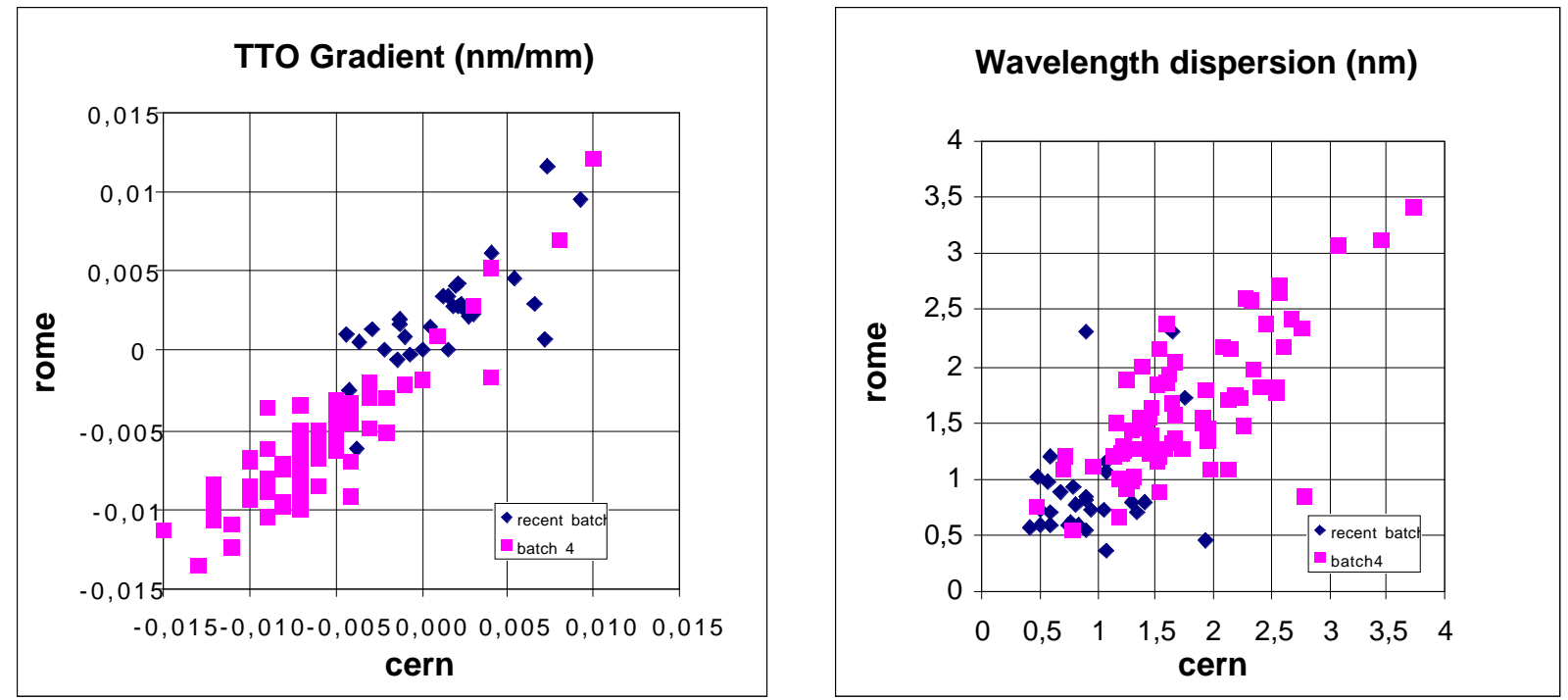

Figure 7: Gradient of the transversal transmission and wavelength dispersion, comparison of the CERN and Rome data on two different sets of crystals. 


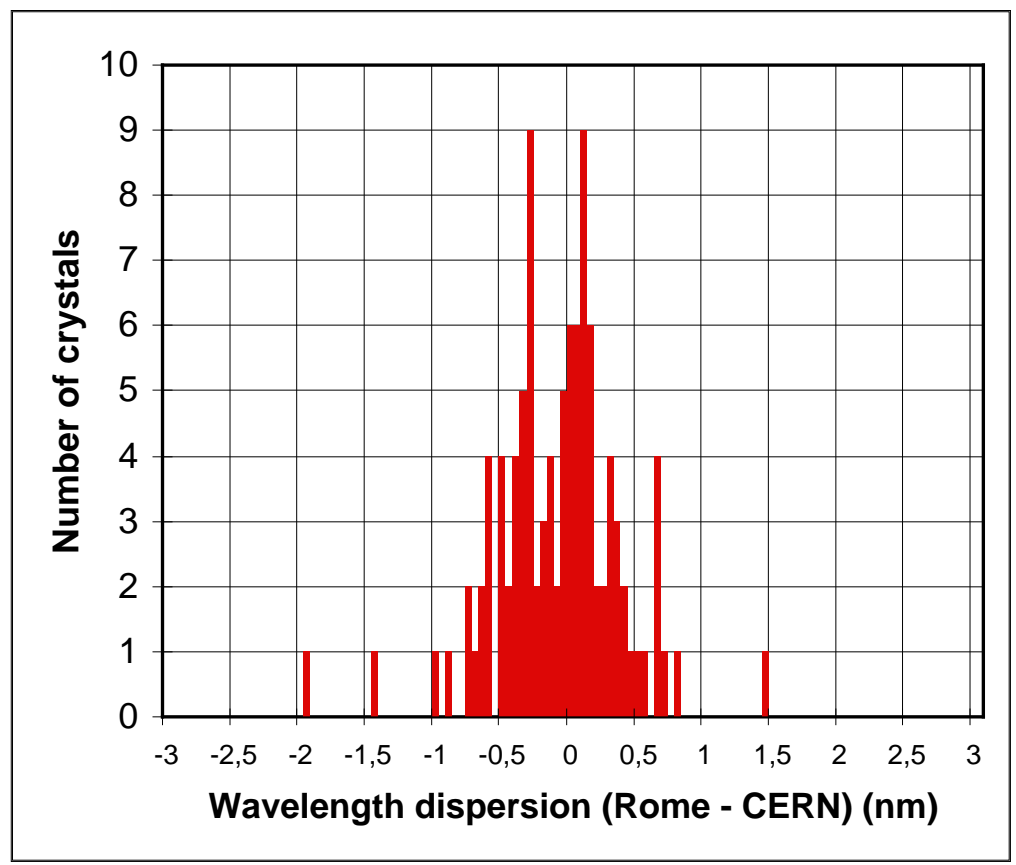

Figure 8: Difference between the Transverse Transmission wavelength dispersion measured by the Rome and the CERN Accos machine.

\section{Conclusions}

After an in-depth comparison of the methods, careful checks and several exchanges of samples, we find satisfactory agreement between the measurements performed on crystals by the two ACCOS machines. Crosschecks will continue until the end of the crystal delivery, in order to ensure the same quality of data. All these comparisons have lead us to establish a protocol for crystal acceptance which is summarized in Ref. [14].

\section{$7 \quad$ Acknowledgements}

We wish to acknowledge the excellent work of all technicians of Lab. 27 at CERN and of the INFN Laboratory in Rome for the successful operation of the ACCOS machines and of the regional centers. We wish to thank the CRISTAL team for their kind collaboration.

The Rome group wishes to thank the ENEA Casaccia laboratory for their hospitality.

The CERN group is grateful to the Minsk team for the excellent collaboration on the ACCOS machines. We wish to thank Francesca Nessi for her comments about this note. 


\section{References}

[1] "CMS The Compact Muon Solenoid", Technical Proposal, CERN/LHCC 94-38, CERN, Geneva Switzerland.

[2] "The Electromagnetic Calorimeter Project", Technical Design Report, CERN/LHCC 97-33.

[3] CMS Note 1999/062, G.Yu. Drobychev et al., "Results of PWO Radiation Hardness Optimization"1

[4] CMS Note 1997/033, G. Basti et al., "A proposal for an Automatic Crystal Control System".

[5] CMS Note 1997/036, G. Drobychev et al., "Studies and proposal for an Automatic Crystal Control System".

[6] S. Baccaro et al., "An automatic device for the quality control of large-scale crystal's production", Nucl. Inst. And Meth. A 459 (2001) 278.

[7] E. Auffray et al., "Performance of ACCOS, an automatic crystal quality control system for the PWO crystals of the CMS calorimeter", Nucl. Inst. And Meth. A 456 (2001) 325.

[8] CMS Note 1996/003, J-M. Le Goff et al., "C.R.I.S.T.A.L. / Concurrent Repository and Information System for Tracking Assembly and production Lifecycles - A data capture and production management tool for the assembly and construction of the CMS and ECAL detector".

[9] S. Baccaro et al., "Precise determination of the light yield of scintillating crystals", Nucl. Inst. And Meth. A 385 (1997) 69.

[10] CMS Note 2001/004, E. Auffray et al., "Development of a uniformization procedure for the $\mathrm{PbWO}_{4}$ crystals of the CMS electromagnetic calorimeter.

[11] CMS Note 2001/033, E. Auffray et al., "Test-beam Results on the Performance of Two Matrices of PbWO4 Crystals for the CMS ECAL and Comparison with Laboratory Measurements ".

[12] CMS Note 1998/038, E. Auffray et al., "Specifications for Lead Tungstate Crystals Preproduction:.

[13] CMS Note 2000/015, M. Montecchi, "Proposal of a functional representation of the PWO transmittance spectrum".

[14] Specification for the PWO crystals in the CMS ECAL Calorimeter, M.Diemoz, (available on http://edh.cern.ch).

CMS Notes are available at the Internet address: http://cmsdoc.cern.ch/cms.html 